SOler $O$, Sinimbu AV, FigueIREDO DC, VIEIRA HKS, Galucio NCR, Pinheiro PNQ, Sena SSI, Silva VG, VIEIRA VMB, LEDO Y
A REORIENTACÃO DA ASSISTÊNCIA FARMACÊUTICA NA SECRETARIA MUNICIPAL DE SAÚDE DE BELÉM (PA): RELATO DE EXPERIÊNCIA

\title{
A REORIENTAÇÃO DA ASSISTÊNCIA FARMACÊUTICA NA SECRETARIA MUNICIPAL DE SAÚDE DE BELÉM (PA): RELATO DE EXPERIÊNCIA
}

The reorientation of pharmaceutical assistance at the department of health of the Belém (PA): experience report

Reorientación de la asistencia farmacéutica en la secretaria municipal de salud de Belém (PA):

relato de experiencia

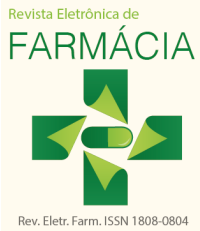

Orenzio Soler ${ }^{1}$, Aline Vilar Sinimbu², Danyelle Costa de Figueiredo², Haila Kelli dos Santos Vieira ${ }^{2}$, Natasha Costa da Rocha Galucio², Priscila de Nazaré Quaresma Pinheiro², Samuel Silva Ibrahim Sena², Valdenira Gonçalves da Silva², Vanja Margareth Bahia Vieira², Yranilde Ledo ${ }^{2 *}$

1 Faculdade de Farmácia. Instituto de Ciências da Saúde, Universidade Federal do Pará (UFPA)

2 Secretaria Municipal de Saúde de Belém, Pará.

*E-mail: yranildeledo@yahoo.com.br

\section{RESUMO}

Introdução: A Assistência Farmacêutica é um conjunto de ações voltadas à promoção, proteção e recuperação da saúde, tanto individual como coletiva, tendo o medicamento como insumo essencial e visando o acesso e ao seu uso racional.

Objetivo: O objetivo do trabalho é apresentar o processo pelo qual ocorreu a reorientação da assistência farmacêutica no município de Belém, Pará (PA).

Métodos: Trata-se de um relato de experiência ocorrido entre setembro de 2014 e agosto de 2015, fundamentado na pesquisa-ação.

Resultados: Tem-se as reflexões sobre as práticas de governança relacionadas à Coordenação Técnica de Assistência Farmacêutica, as ações quanto ao Planejamento Estratégico Situacional e os desfechos esperados com a reorientação da Assistência Farmacêutica pautados na gestão por resultados.

Conclusão: A Coordenação Técnica de Assistência Farmacêutica apresentou uma proposta de reestruturação da Assistência Farmacêutica para o município de Belém; necessitando, ainda, aprimorar sua práxis quanto às práticas de governança.

Palavras-chave: Governança. Planejamento Estratégico. Assistência Farmacêutica.

\section{ABSTRACT}

Introduction: Pharmaceutical Assistance is a set of actions aimed at the promotion, protection and recovery of health, both individual and collective, taking the drug as an essential input and aiming for access and rational use.

Objective: The objective of this work is to present the process by which the reorientation of pharmaceutical assistance occurred in the city of Belém (PA).

Methods: This is an experience report that occurred between september 2014 and august 2015, based on action research.

Results: Reflections on the governance practices related to the Technical Coordination of Pharmaceutical Assistance, the actions regarding Strategic Situational Planning and the expected outcomes with the reorientation of Pharmaceutical Assistance based on management by results.

Conclusion: The Technical Coordination of Pharmaceutical Assistance presented a proposal for the Restructuring of Pharmaceutical Assistance for the city of Belém (PA); as well as to improve its praxis regarding governance practices.

Keywords: Governance. Strategic Planning. Pharmaceutical Assistance. 


\section{RESUMEN}

Introducción: La Asistencia Farmacéutica es un conjunto de acciones dirigidas a la promoción, protección y recuperación de la salud, tanto individual como colectiva, teniendo el medicamento como insumo esencial y visando su acceso y uso racional.

Objetivo: El objetivo del trabajo es presentar el proceso a través del cual ocurrió la reorientación de la asistencia farmacéutica en el municipio de Belém (PA).

Métodos: Se trata de un relato de experiencia ocurrido entre septiembre de 2014 y agosto de 2015, fundamentado en la investigación-acción.

Resultados: Se obtienen las reflexiones sobre las prácticas de gobernanza relacionadas a la Coordinación Técnica de Asistencia Farmacéutica, las acciones sobre la Planificación Estratégica Situacional y los resultados esperados con la reorientación de la Asistencia Farmacéutica pautados en la gestión por resultados.

Conclusión: La Coordinación Técnica de Asistencia Farmacéutica presentó una propuesta de reestructuración de la Asistencia Farmacéutica para el municipio de Belém (PA); necesitando, aún, mejorar su praxis sobre las prácticas de gobernanza.

Palabras clave: Gobierno. Planificación Estratégica. Asistencia farmacéutica.

\section{INTRODUÇÃO}

A Assistência Farmacêutica, uma política pública, tem seus fundamentos jurídicos na Constituição Federal de $1988^{(1)}$, cujo art. 196 assegurou a todos os cidadãos o direito à saúde, estabelecendo o dever do Estado em assegurar tal direito. Com esse desiderato, são formuladas e implementadas políticas sociais e econômicas, que visam reduzir o risco de doenças e garantir o acesso universal e igualitário às ações e aos serviços voltados para a promoção, proteção e recuperação da saúde.

Esse dispositivo constitucional foi regulamentado pela Lei nº 8.080/1990 (Lei Orgânica da Saúde), cujo art. 6 inseriu a formulação da política de medicamentos no campo de atuação do Sistema Único de Saúde (SUS) e atribuiu ao setor da saúde a responsabilidade pela "[...] execução de ações de assistência terapêutica integral, inclusive farmacêutica." (2)

A partir de uma construção normativa da Comissão Intergestores Tripartite (CIT): Ministério da Saúde (MS); Conselho Nacional de Secretários Estaduais de Saúde (CONASS); Conselho Nacional de Secretários Municipais de Saúde (CONASEMS) e do Conselho Nacional de Saúde (CNS), foi articulada a regulamentação a Política Nacional de Medicamentos (PNM) por meio da Portaria GM n³.916, de 30 de outubro de 1998 (3). Essa política tem como propósito garantir segurança, eficácia e qualidade dos medicamentos, a promoção do uso racional e o acesso da população àqueles medicamentos considerados essenciais.

Além disso, o Conselho Nacional de Saúde (CNS) aprovou a Política Nacional de Assistência Farmacêutica por meio da Resolução $n^{\circ}$ 338, de 6 de maio de 2004. ${ }^{(4)}$ Essa resolução estabeleceu um conjunto de ações voltadas à promoção, proteção e recuperação da saúde individual e coletiva, tendo o medicamento como insumo essencial e visando ao seu acesso e uso racional. Registra-se, que a garantia do acesso equitativo às ações de saúde inclui, necessariamente, a Assistência Farmacêutica.

\section{CONTEXTO DA ASSISTÊNCIA FARMACÊUTICA EM BELÉM EM 2014}

A auditoria realizada pelo Tribunal de Contas da União (TCU n 025.954/2014-8) sobre a gestão da Assistência Farmacêutica Básica (AFB) em Belém (PA), tendo como recorte temporal o período entre janeiro de 2013 e setembro de 2014 , analisou o ciclo da AFB nas etapas de seleção, programação, aquisição, armazenamento, distribuição e dispensação; fundamentados no referencial de planejamento e execução formalizados no âmbito da Comissão Intergestores Bipartite do Pará (CIB-PA). ${ }^{(5)}$ 
Para a auditoria foram formuladas as seguintes questões: 1) Os instrumentos de planejamento preconizados pelo SUS abrangem adequadamente a política de assistência farmacêutica e o Governo do Estado do Pará exerce a coordenação dessa política? 2) A seleção, programação e aquisição de medicamentos do componente básico garantem o atendimento adequado e tempestivo da demanda nas Unidades Básicas de Saúde (UBS), sendo processados de forma transparente? 3) As instalações físicas, os controles da unidade armazenadora e os meios de transporte até as UBS garantem a segurança e a qualidade (integridade) na guarda e entrega de medicamentos e evitam desvios e desperdícios? 4) O recebimento dos medicamentos pelas UBS ocorre tempestivamente e nas quantidades solicitadas, e as condições das instalações físicas, de armazenamento, de controle de estoque e de dispensação são adequadas?

Como resultado, a equipe de auditoria apontou a existência das seguintes falhas e irregularidades(5):

- Não integralização da contrapartida estadual do componente básico da assistência farmacêutica;

- Integralização irregular da contrapartida municipal;

- Ausência de controle ou controle deficiente do estoque de medicamentos na Central de Abastecimento; em Belém chamado de Departamento de Recursos Materiais (DRM) e das Farmácias das UBS;

- Funcionamento inadequado do Sistema Nacional de Gestão da Assistência Farmacêutica (Hórus);

- Estrutura física inadequada do DRM e das farmácias das UBS, comprometendo a qualidade dos produtos estocados;

- $\quad$ Falta de medicamentos nas UBS;

- Ausência dos Certificados de Regularidade Técnica dos farmacêuticos responsáveis pelo DRM e pelas farmácias das UBS;

- Controle insatisfatório de temperatura e umidade dos ambientes e das geladeiras onde estão armazenados os medicamentos nas unidades municipais de saúde.

Após compulsar os autos e com fulcro nesses achados, o TCU concluiu que os recursos da Assistência Farmacêutica não são geridos com eficiência no município de Belém (PA). Consequentemente, o desperdício não é evitado nem está assegurada a oferta tempestiva e suficiente de medicamentos nas Unidades Básicas de Saúde.(5)

Registra-se, ainda, que a partir da Auditoria de Natureza Operacional do Tribunal de Contas da União sobre a Assistência Farmacêutica na Secretaria Municipal de Saúde de Belém (PA) foi estabelecido um Acórdão pelos Ministros do Tribunal de Contas da União (Ata n 41/2015 - Plenário; Sessão Ordinária em 14 de outubro de 2015 - Código eletrônico para localização na página do TCU na Internet: AC-2516-41/15-P) para sanar os problemas identificados e encaminhado cópia deste Acórdão, bem como do Relatório e do Voto que o fundamentaram, ao Ministério da Saúde, ao Departamento de Informática do SUS (DATASUS), ao Departamento Nacional de Auditoria do SUS (DENASUS), ao Tribunal de Contas dos Municípios do Estado do Pará, ao Tribunal de Contas do Estado do Pará, ao Observatório Social de Belém (PA), à Associação dos Renais Crônicos do Estado do Pará, à Secretaria de Estado da Saúde Pública do Pará e à Secretaria Municipal de Saúde de Belém (PA); onde a Coordenação Técnica de Assistência Farmacêutica (CTAF) se comprometeu a construir coletivamente uma proposta de reorientação da Assistência Farmacêutica, reflexionando sua práxis a partir dos conceitos de Governança, Planejamento Estratégico Situacional e Gestão por Resultados.

Nesse contexto, o objetivo deste artigo é apresentar o processo pelo qual ocorreu a reorientação da assistência farmacêutica no município de Belém (PA).

\section{METODOLOGIA}

Trata-se de um Relato de Experiência ${ }^{(6,7)}$, sobre o processo de reestruturação da assistência farmacêutica no município de Belém (PA), ocorrido entre setembro de 2014 e agosto de 2015, fundamentado na Pesquisa-Ação(8,9), utilizando-se dos conceitos de Governança, Gestão por Resultados e Planejamento Estratégico Situacional (PES). ${ }^{(10,11,12)}$ 
O Relato de Experiência incorpora registro de situações e eventos, memória oral e escrita, assim como depoimentos relativos à implementação de programas ${ }^{(6,7)}$. Trata-se de uma estratégia na qual a coleta de depoimentos, os relatos de situações e casos relevantes ocorridos em uma determinada realidade (implantação de um programa, por exemplo), tendo por objetivo criar registros que sejam referência para quem for trilhar caminhos semelhantes. ${ }^{(6,7)}$

O Planejamento Estratégico Situacional (PES) apoia-se nas teorias da situação, da produção social e da ação interativa, com as principais características de ser flexível e interativo. ${ }^{(10,11,12)}$ Prioriza, pois, o planejamento criativo, a descentralização dos sistemas de condução e uma abordagem de gestão por objetivos ou operações e, por fim, a alta responsabilidade decorrente do monitoramento e da prestação de contas. Está relacionado com a tomada de decisão, com a análise de riscos, com o seguimento do processo, com a identificação de agentes sociais e econômicos envolvidos.

Fases do Planejamento Estratégico Situacional(10,11,12):

\section{Momento Explicativo.}

1.1 Definição do foco de atuação e identificação dos atores.

1.2 Momento Explicativo - FASE I: Matriz de Priorização de Problemas.

1.3 Momento Explicativo - FASE I: Relação dos Descritores.

1.4 Momento Explicativo - FASE II - Espinha de Peixe.

1.4.1 Problema?

1.4.2 Por que ocorre? (Causas convergentes).

1.4.3 O que gera? (Consequências convergentes).

1.4.5 Imagem-objetivo para o problema identificado.

1.5 Momento Explicativo - FASE III - Revisão do Momento Explicativo.

2 Momento Normativo - Matriz do Momento Normativo.

3 Momento Estratégico - Matriz do Momento Estratégico.

4 Momento Tático-Operacional - Matriz Final do Plano Operativo.

A Coordenação Técnica de Assistência Farmacêutica (CTAF); de caráter técnico e político, está vinculada ao Núcleo de Planejamento em Saúde (NUPS) da Secretaria de Saúde do Município de Belém (SESMA/PMB). É composta por seis farmacêuticos e um administrador. Desenvolve suas atividades articulada com 125 farmacêuticos lotados em Unidades Municipais de Saúde, Unidades de Pronto Atendimento, Hospitais, entre outros serviços.

As oficinas de trabalho foram coordenadas pelo Observatório Farmacêutico da Faculdade de Farmácia do Instituto de Ciências da Saúde da Universidade Federal do Pará, sendo orientadas pelo diálogo participativo - como uma oportunidade de expressar e alinhar as perspectivas de ambos os atores -, em um cenário comum de expectativas de resultados a serem alcançados. Para elaboração dos relatórios, as oficinas foram gravadas e relatadas por um grupo operativo composto de dez farmacêuticos.

A primeira oficina com oito horas de trabalho foi realizada no dia 09 de setembro de 2014, contando com a presença de 65 farmacêuticos (52\%), tendo como tema central: "Fortalecimento da Assistência Farmacêutica em Belém (PA)". Importante registrar que participaram da mesa de abertura as seguintes instituições: Coordenação da Assistência Farmacêutica Básica da Secretaria de Ciência, Tecnologia e Insumos Estratégicos do Ministério da Saúde ((DAF/SCTIE/MS), Conselho Nacional de Secretários de Saúde (CONASS), Conselho Nacional de Secretários Municipais de Saúde (CONASEMS), Conselho de Secretários Municipais de Saúde do Pará (COSEMS-PA), Departamento de Assistência Farmacêutica do Estado do Pará (DEAF/SESPA), Secretaria Municipal de Saúde de Belém (SESMA/PMB), Departamento de Vigilância Sanitária de Belém (DEVISA/SESMA), 
Conselho Federal de Farmácia (CFF), Conselho Regional de Farmácia do Pará (CRF-PA) e Sindicato dos Farmacêuticos do Pará (SinFar).

Esta oficina contou com as seguintes conferências: "Marcos regulatórios e as ferramentas de apoio às Políticas de Medicamentos e Assistência Farmacêutica" (CONASEMS); "Políticas de Medicamentos e Assistência Farmacêutica do Ministério da Saúde, em especial sobre o Qualifar - SUS: Eixo Cuidado" (DAF/SCTIE/MS); "Atual Modelo de Assistência Farmacêutica em Implementação em Curitiba (PR) - Componente Cuidado: Qualifar-SUS - apresentado pela Secretaria Municipal de Saúde de Curitiba (PR). O encerramento foi feito pelo presidente do COSEMS-PA.

A segunda oficina com oito horas de trabalho ocorreu no dia 10 de novembro de 2014, onde se discutiu o "Planejamento Estratégico Situacional e a Reorientação da Assistência Farmacêutica em Belém (PA)", com a participação de 52 farmacêuticos (42\%) selecionados de serviços estratégicos da rede municipal de saúde. Participaram, também, os diretores e coordenadores dos órgãos envolvidos direta e indiretamente com a Assistência Farmacêutica, a exemplo do Departamento de Administração, Departamento Financeiro, Departamento Jurídico, Comissão Permanente de Licitação, Núcleo de Promoção à Saúde, Departamento de Ações em Saúde, Divisão de Recursos e Materiais, Hospitais, Unidades Municipais de Saúde e Casas Especializadas, entre outros observadores.

Partiu-se de indicadores de estrutura, de processos e de resultados ${ }^{(13)}$, para estabelecer a Relação de Problemas e seus respectivos Descritores relacionados a Assistência Farmacêutica, a partir da qual se fez a Matriz de Priorização, em que cada participante emitiu a pontuação para os problemas descritos na matriz. Após a somatória das pontuações atribuídas para cada problema, elegeu-se como principal problema o "Desabastecimento" com 128 pontos, em segundo lugar "A Assistência Farmacêutica não estar regulamentada como um eixo transversal no organograma da SESMA/PMB" com 115 pontos e em terceiro a "Falta de planejamento e harmonização de ações/atividades/tarefas na Assistência Farmacêutica" com 108 pontos.

Foi decidido centrar esforços para a resolução do problema "A Assistência Farmacêutica não estar regulamentada como um eixo transversal no organograma da SESMA/PMB". Acredita-se que a Assistência Farmacêutica deve ser reconhecida como um eixo transversal às ações de promoção, prevenção, paliação, tratamento e cura.

Para o problema foi elaborado o Diagrama de Causa e Efeito (Espinha de peixe), que embasado nos descritores, permitiu definir a imagem-objetivo: "A Assistência Farmacêutica como Departamento no organograma da SESMA/PMB".

A Matriz do Momento Normativo foi desenvolvida a partir da Causa Convergente "Falta de autonomia da Assistência Farmacêutica na Secretaria Municipal de Saúde" e da Consequência Convergente "Aumento dos gastos com os agravos à Saúde", o que possibilitou definir como Objetivo Geral: "Elaborar um Plano Estratégico Situacional para Regulamentar a Assistência Farmacêutica como um Departamento na SESMA/PMB"; proporcionando autonomia e reduzindo custos. A partir das Causas e Consequências Secundárias foram definidos os objetivos específicos, para os quais foram estabelecidas as operações e ações, conforme preconizado pela Matriz do Momento Normativo.

As demais operações e ações do Plano Operativo também foram estabelecidas para a "Reestruturação da Assistência Farmacêutica no Município de Belém" e, tendo sido elaborado o Plano Estratégico Situacional para "Regulamentar a Assistência Farmacêutica como um Departamento na SESMA/PMB", a próxima etapa foi analisar a viabilidade e factibilidade por meio da Matriz do Momento Estratégico.

O Momento Estratégico foi um diferencial do Planejamento Estratégico Situacional (PES), pois trouxe à reflexão as situações descritas no Momento Normativo (o que deve ser), mas, que por algum motivo não estavam em condições de serem executadas, sendo representadas como déficit. Construíram-se mecanismos para viabilizar as ações e operações essenciais para que o Plano Operativo se tornasse exequível.

Para cada objetivo específico foi feita a análise estratégica referente ao problema "A Assistência Farmacêutica não estar regulamentada como um Departamento no organograma da SESMA/PMB" em busca da Imagem-Objetivo: "Estabelecer a Assistência Farmacêutica como Departamento na SESMA", obtendo-se, assim a Matriz do Momento Estratégico. 
Para finalizar o Plano Operativo, estabeleceram-se, por meio do protocolo, os Indicadores de Avaliação e os Indicadores de Monitoramento para o acompanhamento de sua execução. Com os indicadores pactuados foi possível a elaboração da Matriz Final do Plano Operativo com a inclusão das operações e ações estratégicas.

Governança compreende a estrutura (administrativa, política, econômica, social, ambiental, legal e outras) posta em prática para garantir que os resultados pretendidos pelas partes interessadas sejam definidos e alcançados. Pode ser descrita como um sistema pelo qual as organizações são dirigidas, monitoradas e incentivadas, envolvendo os relacionamentos entre sociedade, alta administração, servidores ou colaboradores e órgãos de controle. ${ }^{(10,14)}$

A Gestão por Resultados é um ciclo que começa com o estabelecimento dos resultados almejados, a partir da tradução dos objetivos de governo, assegurando o monitoramento e a avaliação do desempenho da organização (política pública) a partir do alcance desses resultados, retroalimentando o sistema de gestão e propiciando ações corretivas decorrentes dessa avaliação. $(15,16)$ Caracteriza-se como uma das etapas da formulação da política pública, traduzindo ações e objetivos orientados em resultados mensuráveis; propiciando eficiência e accountability.

Quanto aos indicadores relacionados às práticas de governança, optou-se por seguir o modelo apresentado no Referencial Básico de Governança do Tribunal de Contas da União.(10) Os resultados estão apresentados na forma dicotômica de "sim" e "não", sendo descritos quanto ao cumprimento ou não das seguintes práticas: pessoas e competências; princípios e comportamentos; liderança organizacional; relacionamento com partes interessadas; estratégia organizacional; alinhamento transorganizacional; estrutura de governança; gestão de riscos e controle interno; auditoria interna; accountability e transparência

Esta pesquisa-ação foi registrada na Plataforma Brasil (SISNEP) sob Certificado de Apresentação para Apreciação Ética (CAAE) n 45682315.6.0000.0018 e aprovada conforme os termos da Resolução do CNS nº 466 de 12 de dezembro de 2012.

\section{RESULTADOS E DISCUSSÃO}

\section{Sobre os desfechos do planejamento}

No Quadro 1 estão descritos os Problemas Identificados no nível da Coordenação Técnica de Assistência Farmacêutica (CTAF), assim como os resultados dos encaminhamentos para as resoluções dos mesmos no final do ano de 2015.

Após o esclarecimento e a sensibilização da Secretaria Municipal de Saúde quanto à necessidade de um novo olhar sobre a Assistência Farmacêutica, foram contratados 25 novos farmacêuticos visando readequar-se às necessidades dos órgãos reguladores, no que refere as unidades de saúde, hospitais municipais, unidades de pronto atendimento, central de abastecimento farmacêutico e comissão permanente de licitação.

Para cumprir bem sua função, reconhece-se que a CTAF deve possuir os recursos adequados e o capital humano necessário de modo a atuar com eficiência em benefício da sociedade. ${ }^{(21)}$ Para isso é importante traçar claramente seus objetivos, definir sua estratégia de atuação e adotar ferramentas capazes de orientar as ações de melhoria.

A CTAF deve ser transparente quanto a sua organização e sua visão de futuro. Articular-se de modo propositivo com seus ambientes interno e externo, visando cumprir sua missão institucional. Formular suas estratégias, desdobrá-las em planos de ações e acompanhar sua implementação, oferecendo os meios necessários ao alcance dos objetivos institucionais e à maximização dos resultados. ${ }^{(21,22,23,24)}$

Para atender sua finalidade, o de garantir o acesso e o uso racional de medicamentos seguros e com qualidade, a CTAF precisa ser capaz de coordenar múltiplos atores políticos, administrativos, econômicos e sociais. Nesse sentido, é importante manter a coerência e o alinhamento de estratégias e objetivos entre as organizações envolvidas; institucionalizar mecanismos de 
comunicação, colaboração e articulação entre todos os atores envolvidos, e regular suas ações no campo da gestão técnica da assistência farmacêutica e gestão clínica do medicamento.

Há a necessidade de se estabelecer um efetivo processo de comunicação entre as partes interessadas, visando harmonizar as contrapartidas financeiras entre os entes federativos; aprimorar os componentes da assistência farmacêutica e promover a sua regularização frente aos órgãos de regulação técnica e sanitária.

Quadro 1. Lista de problemas identificados e seus desfechos

\begin{tabular}{|c|c|c|}
\hline $\mathbf{N}^{\circ}$ & PROBLEMA IDENTIFICADO & $\begin{array}{l}\text { DESFECHO EM } \\
\text { DEZEMBRO DE } 2015\end{array}$ \\
\hline 01 & $\begin{array}{l}\text { A Assistência Farmacêutica não estar regulamentada como um departamento no } \\
\text { organograma da SESMA/PMB. }\end{array}$ & Pactuado \\
\hline 02 & Desabastecimento de medicamentos, insumos e material hospitalar. & Correção em curso \\
\hline 03 & $\begin{array}{l}\text { Falta de planejamento e harmonização de ações/ atividades/ tarefas na Assistência } \\
\text { Farmacêutica. }\end{array}$ & Pactuado \\
\hline 04 & $\begin{array}{l}\text { Falta de infraestrutura das farmácias, almoxarifados e CAF para Boas Práticas de } \\
\text { Abastecimento e Distribuição. }\end{array}$ & Correção em curso \\
\hline 05 & Internet deficiente e falta de rapidez no suporte para a utilização do Sistema Hórus. & Correção em curso \\
\hline 06 & Dificuldade na comunicação interna dentro da própria Secretaria de Saúde. & Pactuado \\
\hline 07 & $\begin{array}{l}\text { Falta de coparticipação efetiva da Assistência Farmacêutica no Núcleo de Planejamento } \\
\text { da SESMA. }\end{array}$ & Pactuado \\
\hline 08 & Falta de Gestão do Cuidado (Farmácia Clínica) nos Equipamentos de Saúde. & Correção em curso \\
\hline 09 & $\begin{array}{l}\text { Ausência da participação da DDM (DEVISA) no projeto de construção/ reforma e novos } \\
\text { serviços de unidades. }\end{array}$ & Pactuado \\
\hline 10 & Falta da valorização profissional (Ausência de plano de cargos e salários). & Pactuado \\
\hline 11 & $\begin{array}{l}\text { Desarticulação da padronização de medicamentos de acordo com a tipologia de } \\
\text { atendimento baseado nas fontes de financiamento. }\end{array}$ & Correção em curso \\
\hline 12 & Falta de farmacêutico nos processos de licitação. & Resolvido \\
\hline 13 & $\begin{array}{l}\text { Falta da regularização dos equipamentos de saúde da rede municipal perante aos órgãos } \\
\text { fiscalizadores (Certificações de Boas Práticas). }\end{array}$ & Correção em curso \\
\hline 14 & Dificuldade no andamento dos processos licitatórios entre os setores envolvidos. & Correção em curso \\
\hline 15 & Unidades Municipais de Saúde e Estratégias Saúde da Família sem farmacêuticos. & Correção em curso \\
\hline 16 & Ausência de dados e informação de consumo real para os processos licitatórios. & Correção em curso \\
\hline 17 & Falta de normas e procedimentos (MB e POP e protocolos clínicos). & Correção em curso \\
\hline 18 & $\begin{array}{l}\text { Comprometimento do estoque planejado para rede para atendimento de demanda } \\
\text { judicial e ações não planejadas. }\end{array}$ & Correção em curso \\
\hline 19 & Ausência de reuniões técnicas e políticas periódicas. & Pactuado \\
\hline 20 & $\begin{array}{l}\text { Ausência de controle no armazenamento e dispensação de medicamentos nas } \\
\text { Estratégias Saúde da Família. }\end{array}$ & Correção em curso \\
\hline
\end{tabular}




\begin{tabular}{|c|c|c|}
\hline 21 & Falta de revisão do catálogo de materiais. & Correção em curso \\
\hline 22 & $\begin{array}{l}\text { Falta de autonomia de controle do farmacêutico nos equipamentos de saúde (Lei nº } \\
\text { 13.021/2014). }\end{array}$ & Resolvido \\
\hline 23 & Falta de educação continuada/capacitação para todos os níveis envolvidos com a AF. & Correção em curso \\
\hline 24 & Falta de apoio e conhecimento técnico dos gestores. & Pactuado \\
\hline 25 & Falta de harmonização no processo de escrituração de psicotrópicos. & Correção em curso \\
\hline 26 & $\begin{array}{l}\text { Falta de harmonização da organização e processo de trabalho nas Estratégias Saúde da } \\
\text { Família. }\end{array}$ & Pactuado \\
\hline 27 & Ausência de Programa de Gerenciamento de Resíduos Sólidos de Saúde (PGRSS). & Correção em curso \\
\hline 28 & Falta de Recursos Humanos capacitados para o apoio as ações da AF. & Resolvido \\
\hline 29 & Ausência de indicadores e do registro de produção do farmacêutico. & Pactuado \\
\hline 30 & $\begin{array}{l}\text { Desconsideração do conceito de meritocracia para o preenchimento de cargos } \\
\text { administrativos (gestores e gerentes). }\end{array}$ & Pactuado \\
\hline 31 & Falta de articulação inter-regional na Região Metropolitana I. & Correção em curso \\
\hline 32 & Sobrecarga de trabalho dos farmacêuticos. & Correção em curso \\
\hline 33 & Rotatividade de farmacêuticos, o que dificulta a conclusão das ações. & Correção em curso \\
\hline
\end{tabular}

Legenda: Pactuado = Aceito pela Secretaria Municipal de Saúde, mas aguardando aprovação pelas esferas superiores; $E m$ correção = Aceito pela Secretaria Municipal de Saúde e já sendo resolvido diretamente pelos atores envolvidos.

Fonte: A reorientação da Assistência Farmacêutica na Secretaria Municipal de Saúde de Belém (PA), 2015.

\section{SOBRE AS PRÁTICAS DE GOVERNANÇA}

O Quadro 2 apresenta as práticas de governança relacionadas com a Coordenação Técnica de Assistência Farmacêutica (CTAF). A CTAF está vinculada ao Núcleo de Planejamento em Saúde (NUPS) da Secretaria de Saúde do Município de Belém (SESMA/PMB).

Quadro 2. Práticas de Governança no nível da Coordenação Técnica de Assistência Farmacêutica

\begin{tabular}{|l|c|c|}
\hline \multicolumn{1}{|c|}{ PRÁTICAS DE GOVERNANÇA } & Sim & Não \\
\hline \multicolumn{2}{|l|}{ Práticas quanto a pessoas e competências } & $X$ \\
\hline $\begin{array}{l}\text { Utilizam processo transparente e formalizado que oriente a indicação, a seleção e a nomeação de membros } \\
\text { da equipe (gestão operacional). }\end{array}$ & $X$ \\
\hline $\begin{array}{l}\text { Asseguram a adequada capacitação dos membros da equipe (gestão operacional), de modo que as } \\
\text { competências necessárias à execução de suas atividades sejam desenvolvidas. }\end{array}$ & $X$ \\
\hline Estabelecem sistema de avaliação de desempenho dos membros da equipe (gestão operacional). & $X$ \\
\hline $\begin{array}{l}\text { Garantem que o conjunto dos benefícios da equipe seja transparente e adequado para atrair bons } \\
\text { profissionais e estimulá-los a se manterem focados nos resultados organizacionais. }\end{array}$ & $X$ \\
\hline
\end{tabular}




\section{Práticas quanto aos princípios e comportamentos}

Adotam código de ética e conduta formalmente instituído e suficientemente detalhado e claro que defina padrões de comportamento aplicáveis aos membros da equipe (gestão operacional).

Estabelecem mecanismos de controle adequados para evitar que preconceitos, vieses ou conflitos de interesse influenciem as decisões e as ações de membros da equipe (gestão operacional).

Agem de acordo com padrões de comportamento, baseados nos valores e princípios constitucionais, legais e institucionais e no código de ética e conduta adotado, servindo de exemplo para todos.

Contribuem para a boa reputação da organização por meio de boas relações com o cidadão e com outras instituições.

\section{Práticas quanto à liderança organizacional}

Avaliam, direcionam e monitoram a gestão, especialmente o alcance de metas institucionais e o comportamento dos membros da equipe (gestão operacional).

Definem os papéis e distribuem as responsabilidades entre os membros da equipe (gestão operacional), de modo a garantir o balanceamento de poder e a segregação de funções críticas.

Responsabilizam-se, perante as estruturas de governança (internas e externas), pelo estabelecimento de políticas e diretrizes para a gestão da organização e pelo alcance dos resultados previstos.

Avaliam os resultados das atividades de controle e dos trabalhos de auditoria e garanti que sejam adotadas as providências cabíveis.

\section{PRÁTICAS DE GOVERNANÇA}

\section{Práticas quanto ao relacionamento com partes interessadas}

Estabelecem modelo de participação social, no qual se promova o envolvimento da sociedade, dos usuários e demais partes interessadas na definição de prioridades.

Estabelecem, divulgam canais de comunicação e consultam com as diferentes partes interessadas e asseguram sua efetividade, consideradas as características e possibilidades de acesso de cada público-alvo.

Publicitam, para conhecimento de todas as partes interessadas, a estrutura de governança vigente na organização, assim como os papéis e as responsabilidades definidos.

Estabelecem relação objetiva e profissional com a mídia, com outras instituições e com auditores.

Asseguram que decisões, estratégias, planos, ações, serviços e produtos fornecidos atendam ao maior número possível de partes interessadas, de modo balanceado, equitativo, sem permitir a predominância dos interesses de pessoas ou grupos.

\section{Práticas quanto à estratégia organizacional}

Estabelecem modelo de gestão da estratégia que considere aspectos como transparência, comprometimento das partes interessadas e foco em resultados.

Estabelecem modelo de gestão que favoreça o alinhamento de operações à estratégia e possibilite aferir o alcance de benefícios, resultados, objetivos e metas.

Estabelecem as estratégias de ações e as formalizam.

\begin{tabular}{|l|l|l|}
\hline es & & $x$ \\
\hline e & & $x$ \\
\hline na & & $x$ \\
\hline os & $x$ & \\
\hline
\end{tabular}




\begin{tabular}{|c|c|}
\hline Comunicam às partes interessadas as estratégias estabelecidas. & $x$ \\
\hline Monitoram e avaliam a execução das estratégias, os principais indicadores operacionais e os resultados. & $\mathrm{X}$ \\
\hline \multicolumn{2}{|l|}{ Práticas quanto ao alinhamento transorganizacional } \\
\hline $\begin{array}{l}\text { Estabelecem mecanismos de articulação, comunicação e colaboração que permitam alinhar estratégias e } \\
\text { operações dos parceiros, envolvidos em políticas transversais e descentralizadas. }\end{array}$ & $\mathrm{X}$ \\
\hline $\begin{array}{l}\text { Estabelecem, de comum acordo, objetivos coerentes e alinhados entre todos os parceiros envolvidos na } \\
\text { implementação das estratégias, para que os resultados esperados possam ser alcançados. }\end{array}$ & $\mathrm{X}$ \\
\hline \multicolumn{2}{|l|}{ Práticas quanto à estrutura de governança } \\
\hline $\begin{array}{l}\text { Estabelecem e mantêm política de delegação e de reserva de poderes, de forma a assegurar a capacidade } \\
\text { de avaliar, dirigir e monitorar a equipe (gestão operacional). }\end{array}$ & 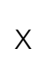 \\
\hline $\begin{array}{l}\text { Definem os papéis e distribuem as responsabilidades entre os membros da equipe (gestão operacional), de } \\
\text { modo a garantir o balanceamento de poder e a segregação de funções críticas. }\end{array}$ & $x$ \\
\hline $\begin{array}{l}\text { Definem, de forma clara, procedimentos e regulamentos afetos a gestão da estrutura interna da equipe } \\
\text { (gestão operacional), bem como os seguintes processos: elaboração, implementação e revisão de políticas; } \\
\text { tomada de decisão, monitoramento e controle. }\end{array}$ & $x$ \\
\hline $\begin{array}{l}\text { Definem instâncias internas de apoio a equipe (gestão operacional) e indicam como elas se relacionam com } \\
\text { as demais estruturas de governança. }\end{array}$ & $X$ \\
\hline
\end{tabular}

\section{PRÁTICAS DE GOVERNANÇA}

\section{Práticas quanto à gestão de riscos e controle interno}

Fomentam a cultura de gestão de riscos como fator essencial para implementar as estratégias, tomar decisões e realizar os objetivos da equipe (gestão operacional).

Estabelecem política e estrutura integrada de gestão de riscos e controle interno.

Asseguram que a gestão de riscos e o controle interno sejam parte integrante dos processos organizacionais da equipe (gestão operacional).

Consideram os riscos que têm impacto sobre outras organizações públicas e demais partes interessadas, comunicam, consultam e compartilham informações regularmente com essas partes.

Monitoram e analisam a gestão de riscos e o sistema de controle interno, a fim de assegurar que sejam eficazes e apoiem o desempenho organizacional da equipe (gestão operacional).

\section{Práticas quanto à auditoria interna}

Estabelecem estatuto que define o propósito, a autoridade e a responsabilidade da auditoria interna.

Proveem condições para que a auditoria interna seja independente e para que os auditores internos sejam proficientes, atuem de forma objetiva e com zelo profissional ao executar seus trabalhos.

Garantem que seja desenvolvido e mantido um programa de garantia de qualidade e melhoria da auditoria interna, compreendendo todos os aspectos da atividade.

Asseguram que a auditoria interna adicione valor à organização. 


\section{Práticas quanto ao accountability e transparência}

Publicam relatórios periódicos de desempenho dos sistemas de governança e de gestão, de acordo com a legislação vigente e com os princípios de accountability.

Publicam, juntamente com os relatórios periódicos, parecer da auditoria interna quanto à confiabilidade das informações prestadas, a regularidade das operações subjacentes e o desempenho das operações.

Publicam a decisão quanto à regularidade das contas proferida pelo órgão de controle externo.

Publicam eventuais avaliações da adequação e do desempenho dos sistemas de governança e de gestão realizadas pelos órgãos de controle externo.

Avaliam, periodicamente, o grau de satisfação das partes interessadas com as estratégias e ações da organização, a satisfação quanto a serviços e produtos fornecidos, assim como avaliam a imagem, a reputação e a confiança do público na organização.

Comprometem-se com a transparência da organização às partes interessadas, admitindo-se o sigilo, como exceção, nos termos da lei.

De ofício, garantem que sejam apurados os fatos com indício de irregularidade ou contrários à política de governança, promovendo a responsabilização em caso de comprovação.

$X$

$x$

Fonte: A reorientação da Assistência Farmacêutica na Secretaria Municipal de Saúde de Belém (PA), 2015.

As práticas de governança e de gestão por resultados são conceitos novos no campo das ações, atividades e tarefas da Coordenação Técnica de Assistência Farmacêutica (CTAF). Uma análise global dessas práticas permite inferir que há muito que apreender.

Quanto a pessoas e competências, sabe-se, que os resultados de uma organização dependem dos Recursos Humanos que nela trabalham. Assim, sendo, a CTAF precisa contar com profissionais que possuam as competências apropriadas. Nessa dimensão da governança, é fundamental mobilizar conhecimentos, habilidades e atitudes dos trabalhadores em prol da otimização dos resultados.

Pires, Menegassi e Tatto ${ }^{(17)}$ registram que o conhecimento tem sido utilizado como um recurso nas organizações de grande relevância e diante disso, a Gestão do Conhecimento através de suas ferramentas têm contribuído para o crescimento em diversos casos. Além da Gestão do Conhecimento, a valorização do Capital Humano também tem aumentado.

Quanto aos princípios e comportamentos a CTAF precisa ter, em seu quadro, pessoas que possuam as competências e as habilidades necessárias, bem como demonstrem elevados padrões de conduta ética. Um dos princípios da boa governança consiste no comprometimento dos membros da equipe com valores éticos, com integridade e com observância e cumprimento da lei.

Pena ${ }^{(18)}$, ao analisar a importância da aplicabilidade das boas práticas de governança no Sistema Único de Saúde (SUS), registra que se trata de um modelo capaz de garantir um atendimento com qualidade aos usuários, garantindo que os serviços prestados sejam eficientes, de custo e qualidade aceitáveis, com transparência na gestão dos recursos públicos.

Quanto à liderança, também chamado de sistema de liderança, que decorre da aplicação dos princípios da coordenação, da delegação de competência e do modelo de governança adotado, constata-se que a CTAF se relaciona com uma complexa estrutura hierárquica para executar os planos em direção ao cumprimento dos objetivos e das metas institucionais.

A CTAF se relaciona diretamente com 29 Unidades Municipais de Saúde (UMS), 10 Centros Especializados (CE), 2 Unidades de Pronto Atendimento (UPA) e 4 Hospitais de Pronto Socorro (HPS). A responsabilidade final pelos resultados produzidos sempre permanece com a autoridade delegante. Por isso, a CTAF deveria ser corresponsável pela definição e avaliação dos 
controles internos que mitigarão o risco de mau uso do poder delegado, devendo ter uma auditoria interna como uma estrutura de apoio comumente utilizada para esse fim.

Quanto ao relacionamento com partes interessadas, onde se observa uma relação direta com o Departamento de Administração (DEAD), Departamento de Gestão e da Regulação do Trabalho em Saúde (DGRTS), Departamento de Ações em Saúde (DEAS), Departamento de Vigilância Sanitária (DEVISA), Departamento de Recursos Materiais (DRM), Assessoria de Comunicação e de Gestão Participativa, Assessoria em Tecnologia da Informação, Assessoria em Gestão do Fundo Municipal de Saúde.

Observa-se, também, uma relação indireta com o Gabinete do Secretário de Saúde (GSS), Departamento de Regulação (DERE), Departamento de Vigilância à Saúde (DEVS), Departamento de Urgência e Emergência (DEUE), Departamento de Gestão e Regulação do Trabalho em Saúde (GRTS), Assessoria Jurídica (NSAJ) e Comissão de Análise de Projetos de Pesquisa (CAPP).

Barbosa, Barbosa e Najberg ${ }^{(19)}$ afirmam que a estruturação e funcionamento da rede de serviços nessa área pode se beneficiar do controle do fluxo e da qualidade de serviços, dependendo da capacidade de uso dos elementos constitutivos do sistema de regulação, com ênfase na sua governança.

Em tempo, Ribeiro e Alcoforado(20), à luz dos mecanismos de governança e gestão pública, de elementos conceituais e da trajetória do debate sobre a nova gerência pública e a Reforma do Estado no Brasil, analisaram o desenho institucional da Secretaria Municipal de Saúde do Rio de Janeiro após as reformas adotadas. Eles concluem que a sustentabilidade desse modelo de gestão implica na capacidade de serem preservadas após a sucessão política.

Ao se considerar o foco necessário da CTAF na prestação de serviços com eficiência, o alinhamento de suas ações com as expectativas das partes interessadas (diretas e indiretas), é fundamental para a otimização de resultados. Precisa-se satisfazer uma complexa gama de objetivos políticos, econômicos e sociais, o que a submete a um conjunto de restrições e influências externas, diferentes daquelas enfrentadas por empresas do setor privado. ${ }^{(20)}$

Quanto à estratégia organizacional, a CTAF apresenta uma relação indireta com a Secretaria Municipal de Assuntos Jurídicos (SEMAJ), Secretaria Municipal de Gestão e Planejamento (SEGEP), Secretaria Municipal de Administração (SEMAD), Secretaria Municipal de Educação (SEMEC), Secretaria Municipal de Urbanismo (SEURB), Secretaria Municipal de Saneamento (SESAN), Secretaria Municipal de Finanças (SEFIN), Secretaria Municipal de Esporte, Juventude e Lazer (SEJEL), Secretaria Municipal de Economia (SECON), Secretaria Municipal de Habitação (SEHAB), Secretaria Municipal de Meio Ambiente (SEMMA).

No atual contexto, há que se compreender que a Assistência Farmacêutica é transversal, necessitando um melhor processo de comunicação entre todas as partes - direta e/ou indiretamente -, interessadas.(21)

Quanto ao alinhamento transorganizacional, a CTAF apresenta uma relação direta com os Conselhos Intergestores Regionais (CIR) e o Conselho Municipal de Saúde (CMS) e, indireta com o Conselho Estadual de Saúde (CES) e o Conselho de Secretários Municipais de Saúde (COSEMS-PA).

Importante observar a necessidade de integração horizontal entre as políticas públicas, o que significa que as ações e os objetivos específicos das intervenções empreendidas pelas diversas entidades devem ser alinhados para se reforçarem mutuamente. ${ }^{(22)}$ Nos casos de políticas de natureza transversal, como é o caso da Assistência Farmacêutica, é essencial que haja mecanismos institucionalizados de coordenação, de forma a criar condições para a atuação conjunta e sinérgica, evitando ainda superposições ou esforços mutuamente contraproducentes.

Quanto às práticas relacionadas à estrutura de governança da CTAF, os critérios observados parecem ser positivos. Sabese que as estruturas de governança são responsáveis por definir, implantar e manter em operação o sistema de governança da organização. ${ }^{(14,15,16)}$

Quanto às práticas relacionadas à gestão de riscos e controle interno pela CTAF, os resultados são negativos. Castro(23) registra que Risco é o efeito da incerteza sobre os objetivos da organização, abrangendo eventos positivos, com o potencial 
de agregar valor, e negativos, com o potencial de destruir valor. O desafio da governança para a CTAF é determinar quanto risco aceita na busca do melhor valor para os cidadãos e demais partes interessadas, o que significa prestar serviço de interesse público da melhor maneira possível.(24)

Quanto às práticas relacionadas à auditoria interna, observa-se que a CTAF não as cumpre. A auditoria interna precisa ser implantada para avaliar a eficácia dos controles internos. Trata-se de uma atividade independente e objetiva de avaliação (assurance) e/ou de consultoria, desenhada para adicionar valor e melhorar as operações; a partir da aplicação de uma abordagem sistemática e disciplinada para avaliar e melhorar a eficácia dos processos de gestão de riscos, controle e governança.(23)

Quanto às práticas relacionadas ao accountability e transparência também não são cumpridas pela CTAF. Sabe-se que há o dever de prestar contas de sua atuação e assumir, integralmente, as consequências de seus atos e omissões; incluindo mecanismos de prestação de contas e de responsabilização para garantir a adequada accountability.(14,15,16) Precisa-se de transparência para garantir a efetividade da accountability.

A prestação de contas não deve restringir-se ao desempenho econômico-financeiro, mas contemplar também os demais fatores (inclusive intangíveis) que norteiam a ação gerencial e que conduzem à criação de valor para a organização.(14,15,16) Filgueiras ${ }^{(14)}$ afirma que a governança de sistemas de avaliação de políticas públicas que envolve o Poder Legislativo na função de avaliação é condição para produzir maior efetividade governamental e accountability.

\section{CONCLUSÃo}

A Coordenação Técnica de Assistência Farmacêutica apresentou uma proposta de reestruturação da Assistência Farmacêutica para o município de Belém (PA); necessitando, ainda, aprimorar sua práxis quanto às práticas de governança.

\section{AGRADECIMENTOS}

Coordenação da Assistência Farmacêutica Básica da Secretaria de Ciência, Tecnologia e Insumos Estratégicos do Ministério da Saúde ((DAF/SCTIE/MS); Conselho Nacional de Secretários de Saúde (CONASS); Conselho Nacional de Secretarias Municipais de Saúde (CONASEMS); Conselho de Secretários Municipais de Saúde do Pará (COSEMS-PA); Departamento Estadual de Assistência Farmacêutica do Pará (DEAF/SESPA); Secretaria Municipal de Saúde de Belém (SESMA/PMB); Conselho Federal de Farmácia (CFF); Conselho Regional de Farmácia do Pará (CRF-PA) e Sindicato dos Farmacêuticos do Pará (SinFar).

\section{REFERÊNCIAS}

1. Brasil. Presidência da República. Constituição da República Federativa do Brasil. D.O.U. 191-A DE 05/10/1988, P.1. Brasília. DF.

2. Brasil. Presidência da República. Lei Federal n 8.080, de 19 de setembro de 1990. Dispõe sobre as condições para a promoção, proteção e recuperação da saúde, a organização e o funcionamento dos serviços correspondentes e outras providências. Diário Oficial da União, Brasília, DF, 20 set.1990. Seção I, p.18055-18059.

3. Brasil. Ministério da Saúde. Portaria no 3.916 de 30 de outubro de 1998. Aprova a Política Nacional de Medicamentos. Brasilia: Diário Oficial da União. 1998.

4. Brasil. Conselho Nacional de Saúde. Resolução CNS no 338 de 6 de maio de 2004. Aprova a Política de Assistência Farmacêutica. Brasília: Diário Oficial da União. 
5. Brasil. Tribunal de Contas da União. Auditoria de Natureza Operacional sobre a Assistência Farmacêutica na Secretaria Municipal de Saúde de Belém (PA) - Sesma: TCU nº 025.954/2014-8. Brasília. DF.

6. Minayo MCS. Análise qualitativa: teoria, passos e fidedignidade. Ciênc. Saúde Coletiva, 2012; 17(3): 621-6.

7. Yin RK. Estudo de caso: planejamento e métodos. 5. ed. Bookman: Porto Alegre, RS, Brasil. 2015. ISBN 978-85-8260-232-4

8. Mallmann EM. Pesquisa-ação educacional: preocupação temática, análise e interpretação crítico-reflexiva. Cadernos de Pesquisa, 2015; 45(155): 76-98. http://dx.doi.org/10.1590/198053143088

9. Mucci CBMR, Mafra RM. Pesquisa-ação e mediação dialógica como práticas metodológicas para emergência da ação comunicativa em Conselhos Gestores de Políticas Públicas. Rev. Adm., 2016; 50(1):107-128. DOI: http://dx.doi. org/10.1590/0034-7612136991

10. Brasil. Tribunal de Contas da União. Referencial básico de governança/Tribunal de Contas da União. - Brasília: TCU, Secretaria de Planejamento, Governança e Gestão, Coordenação-Geral de Controle Externo dos Serviços Essenciais ao Estado e das Regiões Sul e Centro-Oeste. 57 p. 2013

11. Freitas BP. Caminhos do Planejamento: alternativas de reflexão e ação na contemporaneidade. Cadernos Zigmunt Bauman. 2015; 5(10):62-81. ISSN: 2236-4099

12. Organização Pan-Americana da Saúde (OPAS). Manual de planejamento, execução e avaliação de projetos da Representação da OPAS/OMS no Brasil / Organização Pan-Americana da Saúde. Brasília: Organização Pan-Americana da Saúde. 2014; 62p.: il. ISBN: 978-85-7967-011-4

13. Azevedo AC. Avaliação de desempenho de serviços de saúde. Revista de Saúde Pública, 1991; 25(1):64-71. https://dx.doi. org/10.1590/S0034-89101991000100013

14. Filgueiras F. Governança de sistemas de avaliação em perspectiva comparada. In: Filgueiras F, Queiroz L. Cadernos Enap, 2016; 47, 107 p. ISSN 0104-7078

15. Gomes EGM. Gestão por Resultados e eficiência na Administração Pública: uma análise à luz da experiência de Minas Gerais. São Paulo: EAESP/FGV, 2009, 187 p. (Tese de Doutorado apresentada ao Curso de Doutorado de Administração Pública e Governo da EAESP/FGV, Área de Concentração: Transformações do Estado e Políticas Públicas).

16. Pará. Governo do Estado do Pará. Gestão por Resultados. Diário Oficial n 32103 de 24 de fevereiro de 2012 . Gabinete do Governador. Decreto $n^{\circ}$ 352, de 23 de fevereiro de 2012. Dispõe sobre a institucionalização do modelo de gestão para resultados no âmbito do Poder Executivo Estadual.

17. Pires LADB, Menegassi CHM, Tatto L. Gestão do conhecimento e capital humano: uma revisão sistemática de literatura. Revista Conbrad, 2016; 1(3): 204-220. ISSN 2525-6815

18. Pena AA. Boas Práticas de Governança e sua Aplicabilidade no Sistema Único de Saúde (SUS). Orientadora: Menelau, S. Monografia apresentada ao Curso de Especialização em Gestão Pública na Saúde. Faculdade de Economia, Administração e Contabilidade. Universidade de Brasília, como requisito parcial para obtenção do título de Especialista em Gestão Pública da Saúde. Brasília. DF. 2016.

19. Barbosa DVS, Barbosa NB, Najberg E. Regulação em Saúde: desafios à governança do SUS. Cad. Saúde Colet., 2016, 24 (1): 49-54 49. DOI: 10.1590/1414-462X201600010106 
20. Ribeiro JM, Alcoforado F. Mecanismos de governança e o desenho institucional da Secretaria de Saúde do Município do Rio de Janeiro (RJ), Brasil. Ciência \& Saúde Coletiva, 2016; 21(5):1339-1349. DOI: 10.1590/1413-81232015215.00342016

21. Ferreira WW, Quintino ND. Planejamento estratégico para qualificação da gestão da assistência farmacêutica: um estudo de caso em um município de pequeno porte em Minas Gerais. Boletim Informativo Geum, 2016; 7(1):93-105. ISSN 2237-7387 (Impresso)

22. Cardoso-Júnior JC. Monitoramento estratégico de políticas públicas: requisitos tecnopolíticos, proposta metodológica e implicações práticas para a alta administração pública brasileira. Texto para discussão/ Instituto de Pesquisa Econômica Aplicada. Rio de Janeiro: Ipea, 2015. ISSN 1415-4765

23. Castro F. A necessidade de alinhamento entre governança corporativa, gestão de riscos e controles internos administrativos para se atingir os objetivos e resultados, agregando valor público. Orientador Prof. Doutor Martins HF. Trabalho de conclusão de curso apresentado como parte dos requisitos para obtenção do título de especialista em Gestão Pública da Escola Nacional de Administração Pública. 2016.

24. Batista FF. (Org.). Experiências internacionais de implementação da gestão do conhecimento no setor público. Rio de Janeiro: Instituto de Pesquisa Econômica Aplicada (Ipea). Ministério do Planejamento, Orçamento e Gestão. 2016. 178. p. ISBN 978-85-7811-266-0. 